\title{
ON THE ASSESSMENT OF THE TEMPORAL EVOLUTION OF GLOBAL TERRESTRIAL REFERENCE FRAMES: THE VEDA APPROACH
}

\author{
Dimitrios AMPATZIDIS
}

BKG German Federal Agency for Cartography and Geodesy, Richard Strauss Allee 11, 60598 Frankfurt a.M. Germany

Corresponding author's e-mail: Dimitrios.Ampatzidis@bkg.bund.de

\begin{tabular}{l} 
ARTICLE INFO \\
\hline Article history: \\
Received 11 September 2018 \\
Accepted 28 January 2019 \\
Available online 15 February 2019
\end{tabular}

Keywords:

Terrestrial reference frame

Helmert transformation

Reference frame assessment

Geometric effect

ITRF2008

DTRF2008

\begin{abstract}
In this paper, we describe an alternative methodology for the assessment of global Terrestrial Reference Frames (TRFs), called the Velocity Decomposition Analysis (VEDA). Although it is related to the well-known Helmert transformation, a new conceptual manner is presented and discussed. All the necessary mathematical formulas for the adjustment and the quality assessment are provided, as well as a discussion of the similarities and differences to the existing approaches. The core of the VEDA concept lays on the separation of the velocities in two parts: the transformation related one and the optimal velocities, respectively. Using the suggested strategy, we test the global TRFs, the ITRF2008 and the DTRF2008. Their comparison in terms of Helmert transformation parameters reveals discrepancies reaching $0.83 \mathrm{~mm} / \mathrm{yr}$ for the orientation rates, $0.97 \mathrm{~mm} / \mathrm{yr}$ for the translation rates and $0.32 \mathrm{~mm} / \mathrm{yr}$ for the scale rate. The comparison between the new approach and the classical Helmert transformation shows a consistency at the level of $0.66 \mathrm{~mm} / \mathrm{yr}$ in a mean sense. In addition, we find a relative bias between the two frames reaching $0.44 \mathrm{~mm} / \mathrm{yr}$. The new approach also allows quantifying the geometric effect which corresponds to the impact of the systematic inconsistencies and the effect of the set of stations global distribution.
\end{abstract}

\section{INTRODUCTION}

Global Terrestrial Reference Frames (TRFs) play a prominent role in precise 4D point determination. Highly accurate and stable TRFs are an indispensable prerequisite for a correct interpretation of geodetic products describing the geometry, the gravity field, and the rotation of the Earth, especially in the framework of the Global Geodetic Observing System (Beutler et al., 2009). The most recent International Terrestrial Reference Frame (ITRF), adopted by the International Earth Rotation and Reference Systems Service (Petit and Luzum, 2010), is the ITRF2014 released on January of 2016 (Altamimi et al., 2016). The previous solution was the ITRF2008 (Altamimi et al., 2011) provided by the National Institute of Geographic and Forest Information (IGN). Alternatively, the DTRF2008 (Seitz et al., 2012) as a realization provided by the German Geodetic Research Institute of the Technical University of Munich (DGFI-TUM) as another official Combination Centre of the (International Earth Rotation and Reference Systems Service, IERS) is available. Another recent realization of a global reference frame is the JTRF2014, provided by the Jet Propulsion Laboratory (JPL, Wu et al., 2015). In general, the ITRFs provided within the last 20 years are based on the combination of the geometric space geodetic techniques: Doppler Orbitography and Radiopositioning Integrated by Satellite (DORIS), Global Navigation Satellite Systems (GNSS), Satellite Laser Ranging (SLR), and Very Long Baseline Interferometry (VLBI); see e.g., Altamimi et al. (2002).

Concerning the accuracy assessment, different possibilities for TRF validation exist. In general, they can be separated into internal and external validation (see Collilieux et al., 2013). The internal validation is implemented through the comparison of the combined solution to each of the technique-wise TRFs. For instance, the ITRF origin consistency is assessed through its comparison with the SLR-based TRF (e.g. Dong and Fang, 2007; Altamimi et al., 2008). The origin is realized from SLR-only with an accuracy of $10 \mathrm{~mm}$ over the time-span of the SLR observations (Altamimi et al., 2011). The global TRF scale consistency can be assessed with the comparison to the VLBI and the SLR based TRFs (Altamimi et al., 2008) since the ITRF2008 scale and its rate agree with the associated scale of VLBI and SLR at the level of $1.05 \mathrm{ppb}$ and $0.05 \mathrm{ppb} / \mathrm{yr}$, respectively (Altamimi et al., 2011). The orientation and its rate are defined through constraining the new ITRF solution having 
the same orientation and orientation rate as the previous one (ITRF2005; Altamimi et al., 2007). In the case of ITRF2008 and DTRF2008 the orientation is realized by applying no-net rotation (NNR, see e.g. Dermanis, 2001) conditions to a number of core stations in a way that there are zero rotations (and rotation rates) between ITRF2008 and ITRF2005 (Altamimi et al., 2011). For ITRF2005, the orientation rate is aligned to ITRF2000 (Altamimi et al., 2007). The orientation rate of ITRF2000 is realized with NNR conditions to the tectonic plate model NNRNUVEL-1A (DeMets et al., 1994). Seitz et al. (2012) realize the NNR conditions for the DTRF2008 with respect to ITRF2005, using a subset of IGS05 stations. Various studies on the consistency of the ITRF orientation rate in comparison to modern plate tectonic models are published (e.g., Altamimi et al., 2003; Kreemer et al., 2006; Altamimi et al., 2012; Kreemer et al., 2014). We should also refer to the approach of the so called kinematic constraints (Altamimi and Dermanis, 2009, 2013) who introduce optimal solutions by imitating the choice within geophysics where one minimizes the relative kinetic energy of all elements of mass within the continuous earth.

A discussion on the external validation is presented by Collilieux and Altamimi (2013). In their study they directly compared the velocity field of a TRF with the velocities from external data sets which are derived from:

a) horizontal plate motion and glacial isostatic adjustment (GIA) based velocities (see also $\mathrm{Wu}$ et al., 2011; Collilieux et al., 2014),

b) time variable gravity

c) sea level variations from tide gauge measurements and altimetry.

An elegant approach regarding the reference frame transformation is given by Chatzinikos and Dermanis (2017). They proved that from any least squares solution (both for coordinates and velocities) on can obtain another least squares solution through the use of the well-known Helmert transformation parameters. We should also refer to Dermanis (2019a) who gives all the necessary theoretical background and formulations for the optimal realization of the reference systems.

In addition to the ITRF assessment, there are numerous investigations for other physical quantities which are closely related to ITRF fundamentals. For instance, the geocenter motion is discussed extensively by many researchers (e.g., Dong, 2003; Collilieux et al., 2009; Fritsche et al., 2010; Argus, 2012). Wu et al. (2012) published some interesting results on the velocity uncertainty between the mass center and the surface of the Earth concerning the translations and the scale rates using the ITRF and technique-based references frames. They offer a consistent theoretical analysis of the different aspects for the geocenter motion for numerous geodetic and geophysical applications (e.g. for reference frames and sea level). Furthermore, they give some examples on geocenter motion applications. It should be mentioned that the ITRS origin is defined in the Center of Mass of Earth System (CM) by the IERS Conventions 2010 (Petit and Luzum, 2010). Wu et al. (2011) estimated $0.5 \mathrm{~mm} / \mathrm{yr}$ origin consistency between ITRF2008 and the CM, using a subset of globally distributed ITRF stations. A discussion on the geocenter definition and on its different approaches is given by Blewitt (2003) who provides all the necessary theoretical background for various geocenter realizations. In addition, he gives sophisticated mathematical formulations for the transformation between the different geocenter definitions. Argus (2007) presents a method for the comparison between the CM and the Center of the Earth (CE), using velocities derived from the four space-geodetic techniques and models for the postglacial rebound. He concludes that the ITRF should be tied to the CE instead of the CM, due to the fact that the CM is poorly constrained from SLR.

The global TRF stability is a crucial factor for its reliable performance. The detailed monitoring of Earth's processes demands a stable and accurate reference frame that can be applied in various scientific directions. For example, the monitoring of sea level change, the precise orbit determination (for GNSS, climate, gravity satellite and altimetric missions), the tectonic plate modeling, the Global Isostatic Models (GIA) investigations and the regional or local geodetic reference systems realization demand a reliable global TRF realization. In this paper we discuss an additional tool for the assessment of the time evolution of global TRFs using the velocity field by the way of the time-dependent Helmert-type transformation. The aim of our effort is to present an alternative concept for the quantification of temporal evolution of the global TRFs in a relative sense, considering not just the origin and the scale but also the orientation rates. We call the new methodology as Velocity Decomposition Analysis (VEDA), because it is based on the separation of the velocities in two parts:

(a) the transformational velocities related to the so called the geometric effect which includes both the various systematic contributions and the effect due to the selected set of stations (global distribution).

(b) the optimal velocities derived from a specified optimality criterion.

The VEDA approach could be considered as a special case of the more general reference frame transformation provided from Chatzinikos and Dermanis (2017). As we already mentioned they give a detailed report on the optimality transformation of a spatio-temporal TRF. On the other hand, here we are focusing on the optimality of the velocities, without taking into account the part of the coordinates optimality procedures.

In advance, the VEDA can serve for quantifying in a relative sense, the geometric effect on the 
estimated velocity field. We provide all the necessary mathematical formulations and comments on some crucial issues for the assessment procedure. The new methodology is applied to the ITRF2008 and the DTRF2008 in order to validate their performances in terms of comparing their temporal evolution.

\section{THEORY}

\subsection{MATHEMATICAL MODEL OF THE VEDA}

The core of the methodology (from now on VEDA) lays on the derivation of a new set of optimal $3 \mathrm{D}$ velocities. This is achievd through the well-known relation of the $3 \mathrm{D}$ Helmert velocity transformation:

$\mathbf{v}^{\prime}=\mathbf{v}_{o p t}+\mathbf{E} \dot{\boldsymbol{\theta}}_{o p t}$

Where $\mathbf{v}^{\prime}=\left[\begin{array}{c}\mathbf{v}_{i}^{\prime} \\ \vdots \\ \mathbf{v}^{\prime}\end{array}\right]$ the vector of the initial $3 \mathrm{D}$ velocities which refers to a specified TRF for the points $i$ to $n, \quad \mathbf{v}_{i}^{\prime}=\left[\begin{array}{lll}v_{x}^{\prime} & v_{y}^{\prime} & v_{z}^{\prime}\end{array}\right]_{i}^{T}$ the triplet of the velocities per axis for an arbitrary point $i$ which also corresponds to the vector of the $3 \mathrm{D}$ position $\mathbf{x}_{i}^{\prime}=\left[\begin{array}{lll}x^{\prime} & y^{\prime} & z^{\prime}\end{array}\right]_{i}^{T}, \mathbf{v}_{\text {opt }}$ the vector of the new optimal $3 \mathrm{D}$ velocities, $\mathbf{E}=\left[\begin{array}{c}\mathbf{E}_{i} \\ \vdots \\ \mathbf{E}_{n}\end{array}\right]$ the design matrix and $\mathbf{E}_{i}=\left[\begin{array}{ccccccc}1 & 0 & 0 & x_{i}^{\prime} & 0 & z_{i}^{\prime} & -y_{i}^{\prime} \\ 0 & 1 & 0 & y_{i}^{\prime} & -z_{i}^{\prime} & 0 & x_{i}^{\prime} \\ 0 & 0 & 1 & z_{i}^{\prime} & y_{i}^{\prime} & -x_{i}^{\prime} & 0\end{array}\right]$ $\dot{\boldsymbol{\theta}}_{\text {opt }}=\left[\begin{array}{lllllll}\dot{T}_{x}^{o p t} & \dot{T}_{y}^{\text {opt }} & \dot{T}_{z}^{\text {opt }} & \delta \dot{S}^{\text {opt }} & \dot{R}_{x}^{\text {opt }} & \dot{R}_{y}^{\text {opt }} & \dot{R}_{z}^{\text {opt }}\end{array}\right]^{T}$

the vector of the optimal Helmert transformation rate parameters ( $T$ corresponds to the origin, $\delta$ s to scale and $\mathrm{R}$ to rotations, respectively).

Applying the Least Squares criterion as follows:

$\mathbf{v}_{\text {opt }}^{\mathbf{T}} \mathbf{C}_{\mathbf{v}^{\prime}}^{-1} \mathbf{v}_{\text {opt }}=\min$ !

where $\mathbf{C}_{\mathbf{v}^{\prime}}$ the error covariance matrix of the initial set of the velocities, we get:

$$
\begin{aligned}
& \dot{\boldsymbol{\theta}}_{\text {opt }}=\left(\mathbf{E}^{T} \mathbf{C}_{\mathbf{v}^{\prime}}^{-1} \mathbf{E}\right)^{-1} \mathbf{E}^{T} \mathbf{C}_{\mathbf{v}^{\prime}}^{-1} \mathbf{v}^{\prime} \\
& \mathbf{v}_{\text {opt }}=\mathbf{v}^{\prime}-\mathbf{E} \dot{\boldsymbol{\theta}}_{\text {opt }}
\end{aligned}
$$

The meaning of the Equation (4) is the separation of the initial velocities into two parts. The first part is related to the geometric effect on the estimated velocities. For simplicity reasons we will call it as Transformation Related Part (TRP) and it is expressed by the term $\mathbf{E} \dot{\boldsymbol{\theta}}_{\text {opt }}$. The geometric effect comprises the (i) impact of numerous systematic effects such as the datum definition constraints and possible undetected biases (ii) the impact of the global distribution of the stations which are involved in the VEDA application (Dermanis, 2015). The geometric effect is expressed in terms of origin, scale, and orientation rates, respectively. The second part $\mathbf{v}_{\text {opt }}$ refers to the optimal velocities. The optimal part implies a set of velocities which they map the real behavior of them, free (or at least quasi-free) from the systematics (as they discussed previously).

The associated covariance matrices will be:

$$
\mathbf{C}_{\dot{\theta}_{\text {opt }}}=\left(\mathbf{E}^{T} \mathbf{C}_{\mathbf{v}^{\prime}}^{-1} \mathbf{E}\right)^{-1}
$$

for the optimal transformation parameters and

$$
\mathbf{C}_{\mathbf{v}_{\text {opt }}}=\mathbf{C}_{\mathbf{v}^{\prime}}-\mathbf{E} \mathbf{C}_{\dot{\boldsymbol{\theta}}_{\text {opt }}} \mathbf{E}^{T}
$$

We should underline that the Gauss-Markov theorem does not apply here. Thus, for our case we are not justified to use the variance factor in order to rescale the associated covariance matrices. It is not the classical error term which is shown in the classical least squares adjustment. Here the minimization criterion does not refer to the random errors, but it refers to velocities. Hence, one must pay attention to the mathematically correct use of the weight matrix. In the Appendix we prove that the inverse of the errors' covariance matrix of the observations minimizes the trace of the covariance matrix of the unknown parameters (Helmert parameters).

The estimated parameter rates just map the geometric effect of each TRF in terms of origin, scale and orientation rates, respectively. They do not have any pure physical meaning, since the VEDA approach should be imposed in a relative sense (we need two different TRFs). These parameters are strongly correlated with the datum definition, i.e. the constraints used for the TRF realization. In addition, they are also dependent on the geometry of the network. Thus, they amalgamate the geometric effect, which is not related to the real dynamic behavior of the network. Any change in the constraints handling or the network's station distribution (e.g. adding or withdrawing stations) will be directly reflected in the parameter estimates. In that sense the VEDA can serve as a diagnostic tool for the assessment of TRF associated temporal evolutions. For instance, one can compare different sets of the estimated parameters implementing different geometries, or one can compare different (International Terrestrial Reference System, ITRS) realizations like ITRF2008 and DTRF2008.

Let us consider the case of two different TRFs $\mathrm{A}$ and $\mathrm{B}$, either implementing different geometries or two fully independent realizations or sharing common points only. According to Equation (4), one can derive the difference of the estimated parameters as: 
Table 1 Comparison between the classical Helmert and the VEDA.

\begin{tabular}{ccc}
\hline & Helmert transformation & VEDA \\
\hline Observations & $\begin{array}{c}\text { Velocity differences of common } \\
\text { stations }\end{array}$ & Sets or subsets of velocities of two TRFs \\
Methodology & $\begin{array}{c}\text { Geometrical connection (spatial } \\
\text { and dynamic) of two reference } \\
\text { frames }\end{array}$ & $\begin{array}{c}\text { Separation of the velocities into two parts: } \\
\text { TRP and the optimal one. In relative sense it } \\
\text { can identify the reference system effects }\end{array}$ \\
TRF assessment & Explicitly defined & Applicable only in a relative sense \\
\hline
\end{tabular}

$$
\begin{aligned}
& \Delta \dot{\boldsymbol{\theta}}_{\text {optA }, B}=\dot{\boldsymbol{\theta}}_{\text {optB }}-\dot{\boldsymbol{\theta}}_{\text {optA }}= \\
& \quad=\left(\mathbf{E}^{T} \mathbf{W}_{B} \mathbf{E}\right)^{-1} \mathbf{E}^{T} \mathbf{W}_{B} \mathbf{v}_{B}-\left(\mathbf{E}^{T} \mathbf{W}_{A} \mathbf{E}\right)^{-1} \mathbf{E}^{T} \mathbf{W}_{A} \mathbf{v}_{A}
\end{aligned}
$$

and the associated covariance matrix:

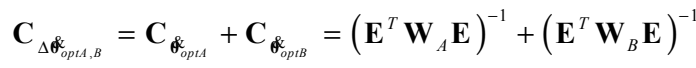

while $\mathbf{W}_{A}=\mathbf{C}_{\mathbf{v}_{\mathrm{A}}}^{-1}$ and $\mathbf{W}_{B}=\mathbf{C}_{\mathbf{v}_{\mathbf{B}}}^{-1}$ are the associated weight matrices, respectively.

The differences of the estimated parameter rates refer to the relative geometric effect between the two frames. The relative geometric effect reflects various systematic inconsistencies which exist between the two frames like different datum constraints handling, undetected biases, differences in the adjustment strategies, and differences in the local ties application. We should again underline the dependence of the results on the selected set of stations for the VEDA application.

\subsection{THE RELATION WITH THE HELMERT SIMILARITY TRANSFORMATION}

The similarity transformation between two reference frames, A and B, yield (e.g. Altamimi et al., 2002; Altamimi and Dermanis, 2012; Dermanis, 2019b):

$\mathbf{v}_{B}=\mathbf{v}_{A}+\mathbf{E} \dot{\boldsymbol{\theta}}_{A \rightarrow B}$

where $\dot{\boldsymbol{\theta}}_{A \rightarrow B}$ the real transformation rate vector connecting the two frames.

Multiplying the two sides of Equation (9) with $\mathbf{E}^{T} \mathbf{W}_{a r b}\left(\mathbf{W}_{a r b}\right.$ a proper arbitrary positive definite weight matrix), we get:

$$
\mathbf{E}^{T} \mathbf{W}_{a r b} \mathbf{V}_{B}=\mathbf{E}^{T} \mathbf{W}_{a r b} \mathbf{v}_{A}+\mathbf{E}^{T} \mathbf{W}_{a r b} \mathbf{E} \dot{\boldsymbol{\theta}}_{A \rightarrow B}
$$

Hence, the real transformation rate parameters will be estimates as follows:

$$
\dot{\boldsymbol{\theta}}_{A \rightarrow B}=\left(\mathbf{E}^{T} \mathbf{W}_{a r b} \mathbf{E}\right)^{-1} \mathbf{E}^{T} \mathbf{W}_{a r b}\left(\mathbf{v}_{B}-\mathbf{v}_{A}\right)
$$

If we compare the Equation (11) with Equation (6) (ibid, VEDA approach) we realize that in the general case, the classical Helmert transformation and the VEDA do not deliver the same results. The comparison between VEDA and Helmert could be realized as follows:

1. Apply the Helmert transformation. Identify and remove the outliers, following a specified criterion (e.g. 3-sigma test).

2. Apply the Helmert transformation and estimate the final seven parameters (rates).

3. Using the same stations, apply for each TRF the VEDA approach. One should pay attention, using the same stochastic model (weights) for both VEDA and Helmert approaches.

4. Compute the parameters difference for each TRF, computed from VEDA.

5. Compare the results between VEDA and Helmert transformation.

\subsection{SOME CONSIDERATIONS REGARDING THE VEDA CONCEPT}

(i) One can raise some questions regarding the theoretical foundation of the VEDA. For example, if we are justified to separate the velocities into a TRP and an optimal part, respectively, using the least squares approach. The separation of the velocities into two parts holds also for other widely-known applications, like the Euler Pole Parameters (EPPs) estimation (e.g. Drewes, 1982). The EPPs define the motion of a plate (the systematic effect) and the residuals which result the inconsistencies of the fitting (the stochastic part). In the case of the VEDA, the optimal part expresses a residual term which is estimated after removing the global trend (geometric effect).

(ii) In addition, we should also notice that the application of the VEDA is inevitably dependent on the selection of the stations. Different selection of the stations will lead to different results. Since the VEDA relies on the relative comparison of two TRFs, it senses the differences with respect to a specific choice of stations set. We can consider different scenarios in order to investigate the pure datum definition and the systematic effects. For example, the VEDA could be applied only to the core stations of the global TRF. The term core hereby refers to the stations that are used for the datum definition of the TRFs. In 
particular, for the stations that are used for the No Net Translation (NNT), No Net Scale (NNS) and No Net Rotation (NNR) conditions (Angermann et al., 2004). If the VEDA is not implemented in the core but in a global mixed set of stations, the associated results will be affected from the differences in datum constraints handling, various systematic inconsistencies, and the network's geometry. That is the reason why we exploit a set of globally distributed stations obeying certain criteria, as we discuss in the Section 3.

(iii) Following the previous comments (i) and (ii), we should mention that the VEDA does not offer any pure geophysical meaning, since the VEDA depends on the set of the selected stations used for its application. The purpose of the VEDA is to stand as an alternative strategy for global TRF assessment. The parameters, the optimal part and the TRP of two TRFs provide information regarding the relative systematic effects between two TRFs (using a common set of points).

(iv) We should also refer to the term alternative. Strictly speaking, the VEDA is based on the classical Helmert transformation through origin, scale, and orientation parameters, respectively. Though, the VEDA concentrates only to the associated parameter rates. The term alternative strategy holds because despite the fact that it estimates the transformation rates (like the Helmert one), we prove that the VEDA does not necessarily give the same results (see discussion in Section 2.2).

(v) The TRP refers to the apparent velocity which depends on the choice of the reference system and it changes with respect to the different reference system options. The constraints are directly dependent on the selection of the geometry of the core stations where are used as the constraints for the reference system definition. These are the so called geometric effects in the velocity estimations. Thus, any change to the geometry of the network (both core and noncore stations) will impact the TRP of the velocities. This could be interpreted as a systematic effect of the velocity estimation.

(vi) The VEDA (like the Helmert transformation) is directly affected from the set of the selected stations, as we mentioned before. The better global distribution, the more reliable results. If the distribution of the stations is not good, it turns out that VEDA results will be also uncertain. However, this is also the case of the classical Helmert transformation. In advance, the geometry plays the same role for both techniques (VEDA and Helmert), since the two techniques share the same design matrix.

(vii) One can also claim that the TRP velocities estimation is strongly dependent from the geometry and this fact could possibly lead to misinterpretation. In any case, the stations contribution is crucial. However, in the VEDA approach we compare two global TRFs (pure relative sense) using the exact same stations and therefore we limit our validation scheme only to a specified geometry.

\section{RESULTS}

\subsection{THE COMPARISON OF ITRF2008 AND \\ DTRF 2008}

The new methodology is applied to the two aforementioned global TRFs: The ITRF2008 (Altamimi et al., 2011) and the DTRF2008 (Seitz et al., 2012). For each global TRF, the coordinates, the velocities and their associated formal errors are published (for ITRF2008 the associated site is: http://itrf.ensg.ign.fr/ITRF_solutions/2008/more_ITR F2008.php, and for the DTRF2008 (Seitz et al., $2012 b)$. The error covariance matrix $\left(\mathbf{C}_{\mathbf{v}^{\prime}}\right)$ for each global TRF refers to the variances of the estimated velocity components and it has diagonal form. For the analysis, stations are excluded if at least one of their standard deviations of the velocity components exceeds $2.0 \mathrm{~mm} / \mathrm{yr}$. The value $2.0 \mathrm{~mm} / \mathrm{yr}$ is empirically chosen as a trade-off between the global TRF's accuracy and its stations distribution. We rejected 145 out of 735 stations after applying this criterion. By choosing smaller values the number of stations would significantly decrease and consequently, the station distribution would become rather sparse. For example, in the case of using $1.0 \mathrm{~mm} / \mathrm{yr}$, we would have one hundred stations less and poorer global distribution. If the value would be enlarged we risk adding unreliable network stations, reducing the quality. Additionally, we apply a classical Helmert transformation in order to detect outliers: any station where at least one of its residual components exceeds three times the residuals standard deviation ( 3 sigma criterion) is rejected. The application of the 3 sigma criterion leads to the removal of 125 stations, out of 590 which fulfill the $2.0 \mathrm{~mm} / \mathrm{yr}$ criterion. Finally, a set of 465 stations is used for our tests. The set comprises 41 VLBI, 18 SLR, 378 GPS, and 28 DORIS stations, respectively. Figure 1 depicts the common stations.

We proceed with the comparison of the two TRFs (ITRF and DTRF2008) in terms of (a) the estimated Helmert parameters and (b) the optimal velocities differences and (c) the TRP velocities.

We choose to use ITRF2008, instead of the actual ITRF2014 (Altamimi et al., 2016), since we want to compare it to the DTRF2008. Till now, the DTRF2014 is not related to any publication or report regarding the official DTRF2014 solution (e.g. the exact procedure of its realization, its consistency, and its validation). Of course, one can follow the same approach for the comparisons between the ITRF2014 and DTRF2014.

We avoid to present any vertical velocities. This fact is dictated due to the not straightforward vertical velocities interpretation, especially for the near-pole regions (which are influenced from the GIA).

\subsubsection{THE APPLICATION OF THE VEDA}

In this section we provide the comparison of the two global TRFs regarding the seven Helmert parameters (differences in translation, scale and 


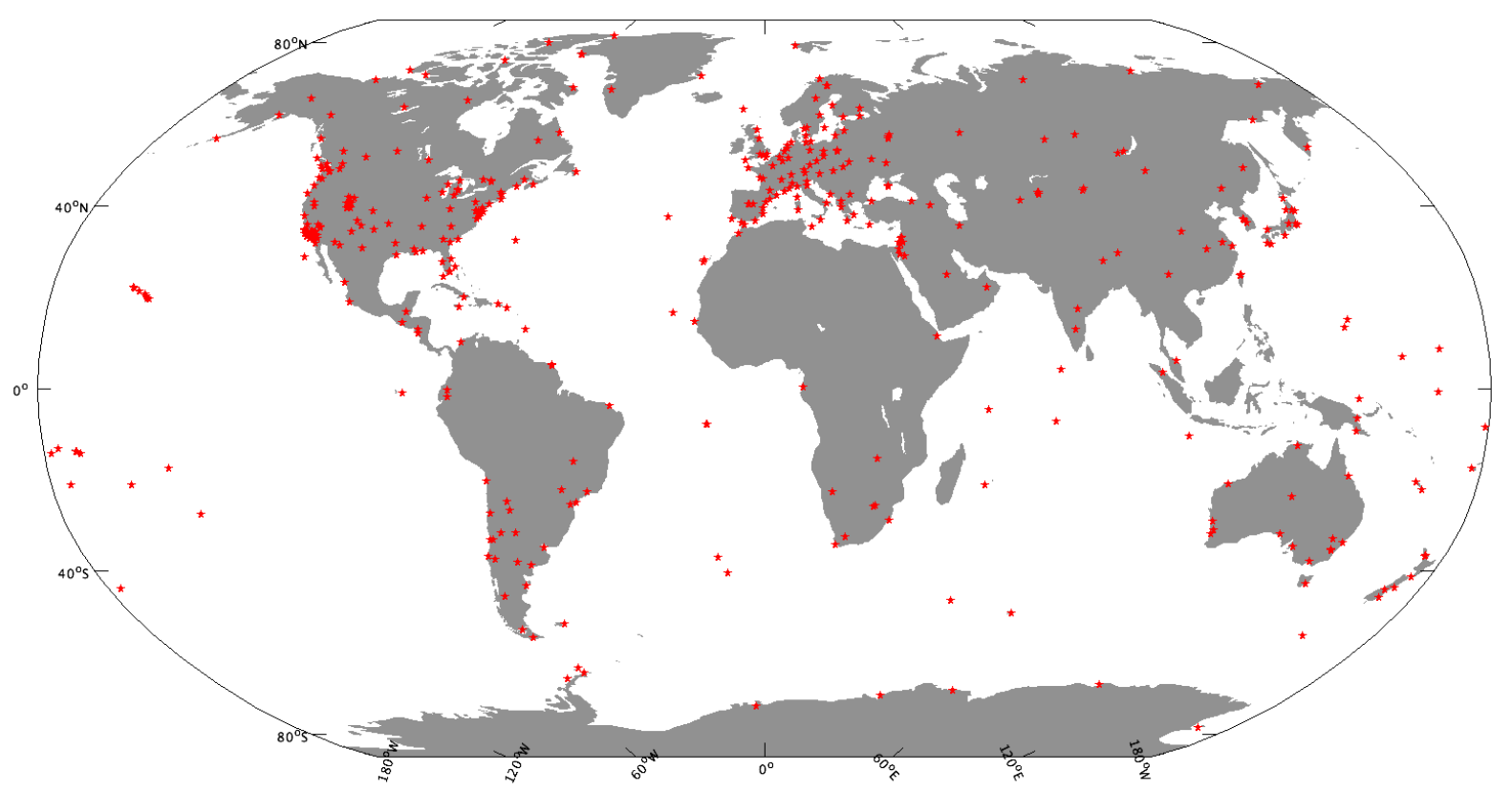

Fig. 1 The 465 common stations (red stars) of ITRF2008 and DTRF2008 fulfilling the $2.0 \mathrm{~mm} / \mathrm{yr}$ accuracy criterion and after removing the outliers.

Table 2 The estimated parameters and their standard deviations of the ITRF2008 and the DTRF2008 for the case of the VEDA and Helmert. All values are in $\mathrm{mm} / \mathrm{yr}$.

\begin{tabular}{|c|c|c|c|c|c|}
\hline \multirow[b]{2}{*}{$\begin{array}{l}\text { estimated } \\
\text { parameter }\end{array}$} & \multicolumn{3}{|c|}{ VEDA } & \multirow[t]{2}{*}{ Helmert } & \multirow{2}{*}{$\begin{array}{c}\text { differences } \\
\text { between VEDA } \\
\text { and Helmert }\end{array}$} \\
\hline & ITRF2008 & DTRF2008 & $\begin{array}{c}\text { differences } \\
\text { (DTRF2008 minus } \\
\text { ITRF2008) } \\
\end{array}$ & & \\
\hline$\dot{T}_{x}$ & $-15.55 \pm 0.02$ & $-14.84 \pm 0.02$ & $0.69 \pm 0.03$ & $0.09 \pm 0.04$ & $0.60 \pm 0.05$ \\
\hline$\dot{T}_{y}$ & $11.35 \pm 0.02$ & $10.38 \pm 0.02$ & $-0.97 \pm 0.03$ & $0.08 \pm 0.04$ & $-1.05 \pm 0.05$ \\
\hline$\dot{T}_{z}$ & $12.05 \pm 0.02$ & $11.12 \pm 0.02$ & $-0.93 \pm 0.03$ & $-0.03 \pm 0.04$ & $-0.92 \pm 0.05$ \\
\hline$d \dot{s}$ & $-0.76 \pm 0.03$ & $-1.08 \pm 0.03$ & $-0.32 \pm 0.04$ & $-0.13 \pm 0.05$ & $-0.19 \pm 0.06$ \\
\hline$\dot{R}_{x}$ & $-4.65 \pm 0.03$ & $-3.82 \pm 0.03$ & $0.83 \pm 0.04$ & $0.00 \pm 0.06$ & $0.83 \pm 0.07$ \\
\hline$\dot{R}_{y}$ & $0.92 \pm 0.03$ & $1.17 \pm 0.03$ & $0.25 \pm 0.04$ & $-0.14 \pm 0.05$ & $0.39 \pm 0.06$ \\
\hline$\dot{R}_{z}$ & $-4.83 \pm 0.03$ & $-4.21 \pm 0.03$ & $0.62 \pm 0.04$ & $0.13 \pm 0.05$ & $0.49 \pm 0.06$ \\
\hline
\end{tabular}

orientation rate, respectively, see Eq. 3 ibid.) which corresponds to the relative geometric effect. Table 2 (columns: 2, 3 and 4) summarizes the estimated parameters together with the differences and their standard deviations for the DTRF2008 and the ITRF2008. The DTRF2008 translation rates are smaller than those of the ITRF2008. The maximum difference is $0.97 \mathrm{~mm} / \mathrm{yr}$ in $y$ direction. The scale rate of the DTRF2008 is estimated $0.32 \mathrm{~mm} / \mathrm{yr}$ larger than that of ITRF2008. For the orientation rates the maximum difference is found for the $x$ axis with $0.83 \mathrm{~mm} / \mathrm{yr}$. We can deduce that the two frames are consistent at the level of $0.66 \mathrm{~mm} / \mathrm{yr}$ with respect to the mean of the differences of the estimates between the frames.

A reason for Helmert related parameters' inconsistencies could arise from different adjustment strategies followed by the two solutions (ITRF2008 employs time series stacking while DTRF2008 uses
Normal Equation stacking). We also observe discrepancies in the orientation rates which could be related to different subsets of points used for NNR realizations. Altamimi et al. (2012) claim that the ITRF2008 NNR definition is probably not better than $2.0 \mathrm{~mm} / \mathrm{yr}$. Seitz et al. (2012) found a disagreement of $0.06 \mathrm{mas} / \mathrm{yr}(\sim 1.9 \mathrm{~mm} / \mathrm{yr})$ between DTRF2008 and the tectonic plate model APKIM2005 (Drewes, 2009). APKIM2005 is a global tectonic plate model which was estimated following the NNR conditions. Finally, these discrepancies could also come from undetected systematic effects occurred in one or both frames. One known reason is the handling of the weights of the local ties added to the equation systems (Seitz et al., 2012). In addition, the selection of local ties is an important factor.

In order to get a relative interpretation of our results we refer to the study of Seitz et al. (2012). They do not directly compare ITRF2008 and 
Table 3 The statistics of the optimal velocities differences between the ITRF2008 (I) and the DTRF2008 (D) of the VEDA. Values are in $\mathrm{mm} / \mathrm{yr}$.

\begin{tabular}{lccc}
\hline Statistics & 3D & horizontal part only & vertical part only \\
\hline Min & -2.85 & -3.77 & -3.06 \\
Max & 3.81 & 2.91 & 3.44 \\
Mean & 0.44 & 1.38 & 0.40 \\
standard deviation & 0.98 & 1.07 & 1.07 \\
\hline
\end{tabular}

DTRF2008. They rather compare each space geodetic technique, individually. In the case of DORIS, a translation discrepancy in the $z$ direction is found between the two global TRFs that reaches $0.8 \mathrm{~mm} / \mathrm{yr}$. The scale difference is estimated at the level of $0.1 \mathrm{~mm} / \mathrm{yr}$ for DORIS, SLR, and VLBI and the orientation differences reach $0.4 \mathrm{~mm} / \mathrm{yr}$ in the case of SLR. At the same time, the RMS of the transformation residuals is 0.98 and $0.82 \mathrm{~mm} / \mathrm{yr}$ for DORIS and SLR, respectively (see Table 18 of (Seitz et al., 2012)). Thereafter, Seitz et al. (2013) published results of the external validation of the DTRF2008. They found that the rate differences between ITRF2008 and DTRF2008 vary from 0.82 for SLR, $0.09 \mathrm{~mm} / \mathrm{yr}$ for VLBI, $0.19 \mathrm{~mm} / \mathrm{yr}$ for GPS, and $0.98 \mathrm{~mm} / \mathrm{yr}$ for DORIS, respectively. We should also consider that Seitz et al. (2013) use 178 stable stations (core stations) in total for their assessment compared to 465 used in our approach. Seitz et al. (2012) claim a velocity consistency between the ITRF2008 and DTRF2008 at the level of $0.5 \mathrm{~mm} / \mathrm{yr}$ while in the present paper we find $0.66 \mathrm{~mm} / \mathrm{yr}$.

By comparing the two global TRFs using all stations instead of separating the space techniques as Seitz et al. (2012) did, we describe only the general behavior of the two realizations. Thus, we do not account for any technique-specific effects or biases, e.g. the scale bias between SLR and VLBI in ITRF2008, which is not visible in the DTRF2008. It is also worth to mention that the results are probably dominated by the space technique with the largest number of contributing stations which is the GPS.

\subsubsection{THE OPTIMAL VELOCITIES DIFFERENCES OF THE TWO GLOBAL TRFS}

Here, we discuss the results of the comparison of the two global TRFs with respect to their differences of the optimal velocities. Table 3 presents the optimal velocities differences between the two frames, according to the VEDA. As we mentioned before these differences can reveal relative systematic effects. We find a mean average value of $0.44 \mathrm{~mm} / \mathrm{yr}$ for the 3D differences, while the horizontal and vertical part is $0.16 \mathrm{~mm} / \mathrm{yr}$ and $0.40 \mathrm{~mm} / \mathrm{yr}$, respectively. Firstly, we see that the vertical part presents a mean average more than two times larger than the horizontal part. If we want to identify the reasons of these results, we should consider the different nature of the velocities. First of all, the up component is less accurately estimated, especially in case of VLBI, GPS and DORIS. For SLR the height is usually at the same quality as the horizontal components. Secondly, while the horizontal velocities are directly related to the tectonic motion globally, the vertical velocities present their own characteristics. For example, they are mainly observed near the Poles, in the Patagonian ice field, and at large parts of North America due to the GIA, see e.g. (Peltier, 1998) and for the rest of the world are much smaller than the horizontal ones. However, since we are dealing with a global TRF these local effects and mainly due to stations distribution can contaminate the final solution.

In advance, the observed relative systematic inconsistencies could be caused by several reasons which however they are not easily identified, such as:

(a) a relative bias due to systematic errors, particularly in the vertical velocities as we discussed before

(b) different adjustment strategies

(c) different bias handling, e.g. different station rejection criteria, different approach in episodic events treatment

(d) differences in local ties application

(e) a combination of both (a), (b), (c) and (d)

Table 3 presents the statistics of the difference between ITRF2008 and DTRF2008, according to the VEDA approach. The mean average of the differences can stand as an indicator of the consistency between the two frames. The closer to zero, the more consistency between the frames. The mean average between could be also interpreted as the bias between the two solutions. In our case, for the 3D optimal velocities there is a deviation of $0.44 \mathrm{~mm}$ between the ITRF2008 and DTRF2008. In advance, the form the differences distribution gives the sense of the errors behavior. A distribution which is close to the normal (Gaussian) one shows a randomness of the errors. On the other hand, the deviation from the normal distribution is a sign of the presence of some systematic effects.

Figure 2 gives the sense of the optimal velocities differences. The distribution of the differences seems to almost follow the normal distribution, slightly 


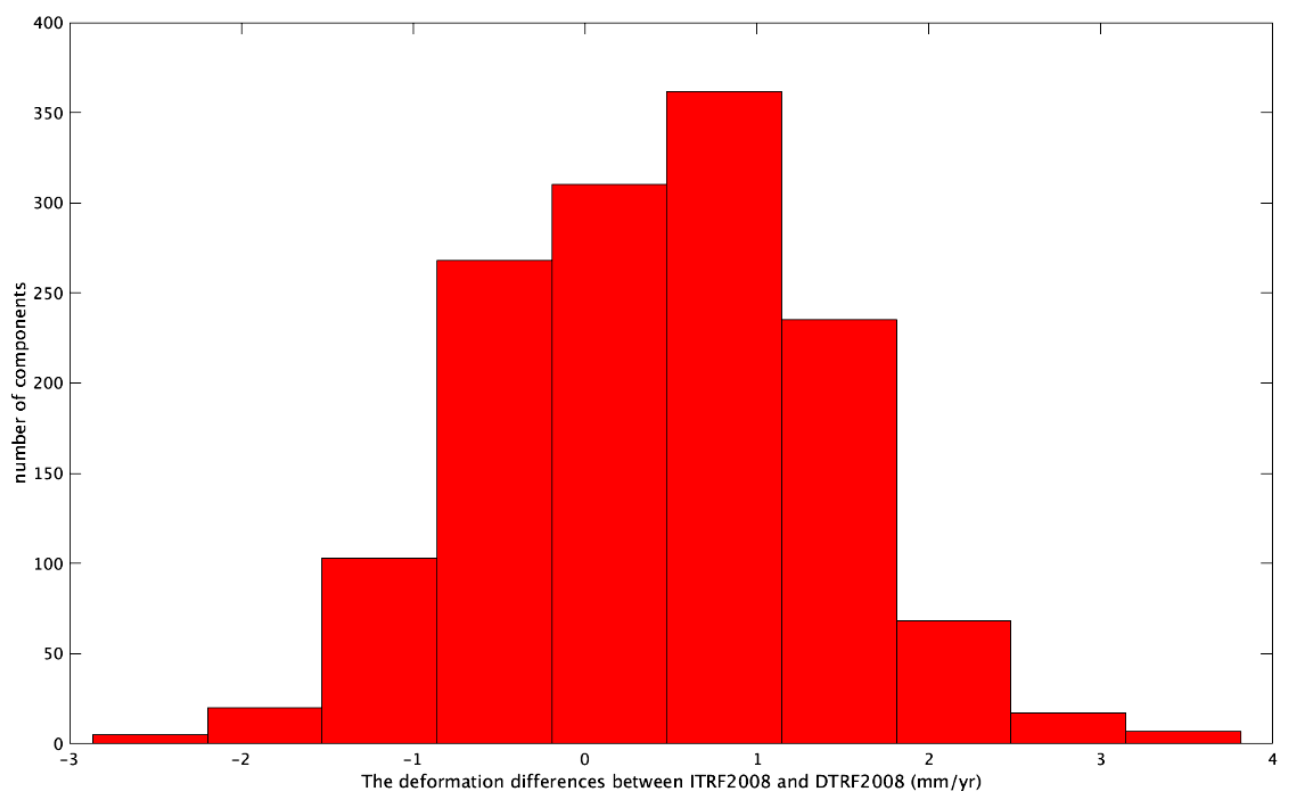

Fig. 2 The optimal velocities differences histogram.

shifted over the mean average of $0.44 \mathrm{~mm} / \mathrm{yr}$. This shift could be interpreted as a relative bias of the estimated optimal velocities differences between the TRFs which should be investigated. It is also rather possible that there are on each TRF solutions some particular systematic effects (except from those described in section 3.1.1) on the velocities which are related to other impacts, e.g. non-linear behavior of the stations, atmospheric loading effects (Altamimi et al., 2016).

Finally, an explanation for the aforementioned inconsistency would be the different combination methodologies followed by the analysis centers. The different combination methodologies could lead to a deviation between the solution shown and interpreted as systematic effect on the velocities

Figures 3 and 4 depict both the TRP and the optimal velocities for ITRF2008 and DTRF2008, respectively.

Figures 3 and 4 reveal that there is a significant deviation between the TRP and the optimal velocities, for both frames, especially for the South Pole stations. In the case of the South Pole, the vector's direction of the TRP velocity is exact opposite from the one of the optimal part. This peculiar behavior seems to be related with the fact that none of the South Pole Stations involved in the NNR implementation for both TRFs. This is also an indication of the dependence of the estimated TRF velocities from the reference system definition, especially for the case of NNR conditions. The TRP velocities show particular regional trends. E.g. almost all of the European, American, African and Australian stations present the same orientation and magnitude, respectively. The optimal velocity computation seems to de-trend the total estimated amount of the TRF velocities.

\subsubsection{THE RESULTS OF THE CLASSICAL HELMERT TRANSFORMATION}

The results of the classical Helmert transformation of DTRF2008 into ITRF2008 are compiled in Table 2 (column 5). The estimated parameters vary from -0.14 to $0.13 \mathrm{~mm} / \mathrm{yr}$. The standard deviations of the estimated parameters are smaller or equal to $0.06 \mathrm{~mm} / \mathrm{yr}$. The velocity residuals RMS is $0.58 \mathrm{~mm} / \mathrm{yr}$. The maximum and the minimum residuals are $1.72 \mathrm{~mm} / \mathrm{yr}$ and $-1.70 \mathrm{~mm} / \mathrm{yr}$, respectively. Table 4 summarizes the statistics of the Helmert transformation residuals. The estimated parameters show differences between the two global TRFs which do not exceed the level of $0.14 \mathrm{~mm} / \mathrm{yr}$ and do not agree with those of Seitz et al. (2012). As we mentioned before, Seitz et al. (2012) do not directly compare the DTRF2008 with ITRF2008, but they compare each technique separately. Moreover in the present study we use almost three times more stations than Seitz et al. (2012) did. Comparing the statistics of the Helmert transformation residuals and those of the VEDA (Table 4) for the optimal velocities differences, we can imply that some relative systematic biases still exist, which the classical Helmert transformation cannot detect. For instance, with the VEDA approach we find a mean average of

Table 4 The statistics of the residuals of the Helmert transformation. Values are in $\mathrm{mm} / \mathrm{yr}$

\begin{tabular}{lc}
\hline statistics & residuals \\
\hline $\min$ & -1.70 \\
$\max$ & 1.72 \\
mean & 0.00 \\
standard deviation & 0.58 \\
\hline
\end{tabular}




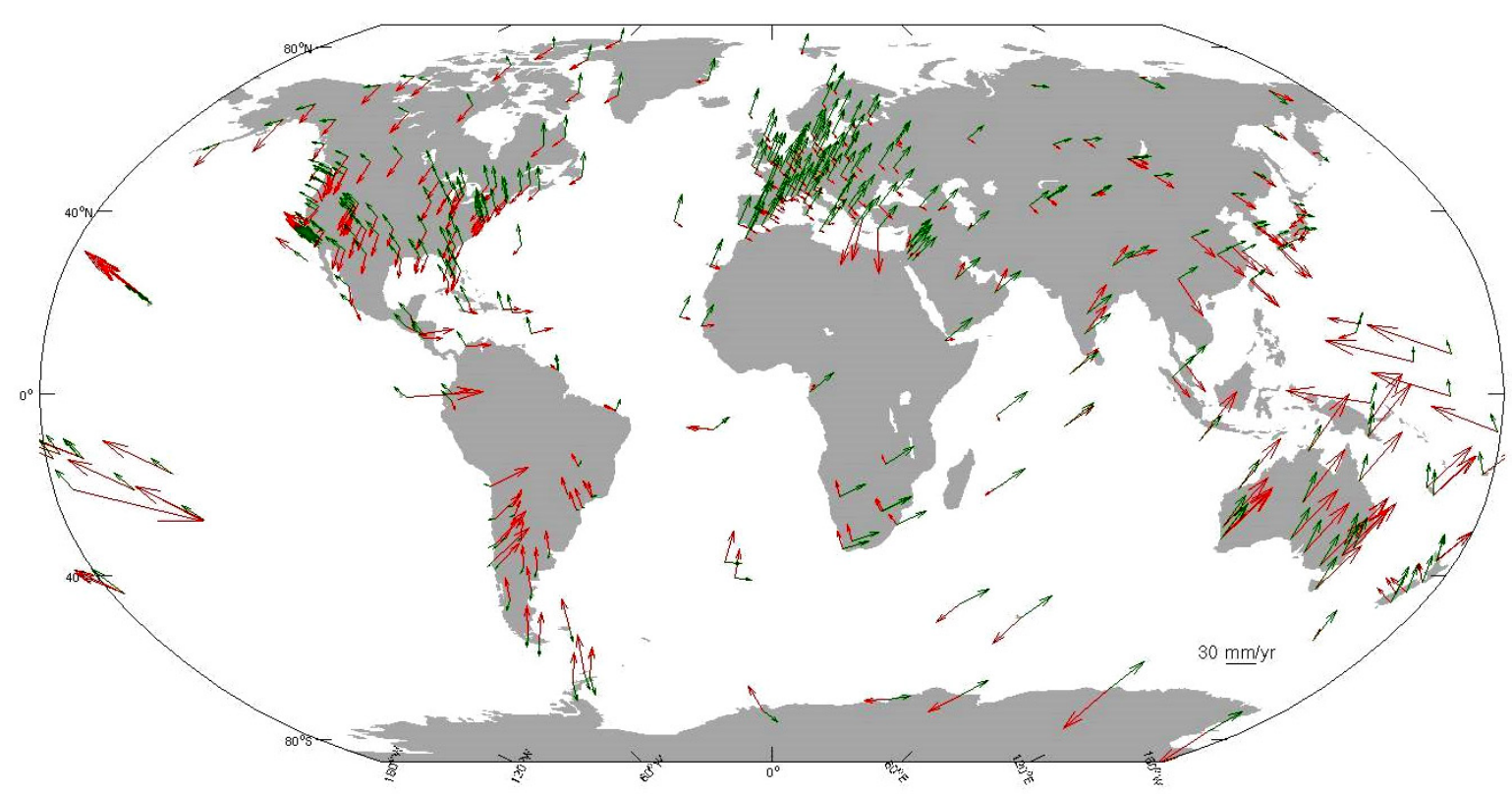

Fig. 3 The TRP and the optimal velocities for ITRF2008. The green thin arrows refer to the TRP and the red thick ones to the optimal velocities.

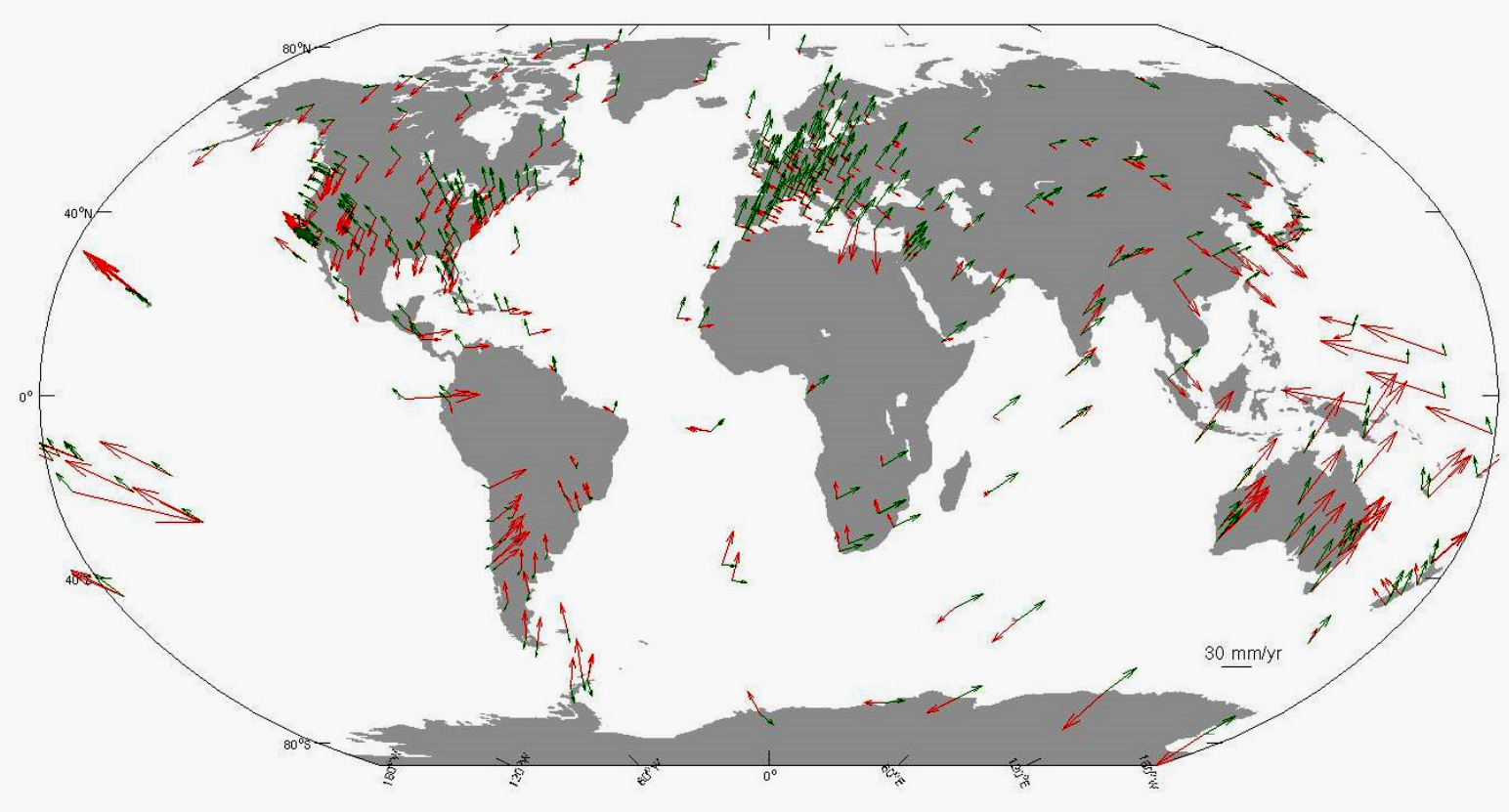

Fig. 4 The TRP and the optimal velocities for DTRF2008. The green thin arrows refer to the TRP and the red thick ones to the optimal velocities.

$0.44 \mathrm{~mm} / \mathrm{yr}$, while in the case of the Helmert transformation is zero.

In Table 2 (column 6) presents the discrepancies and their associated uncertainties between the new approach and the Helmert transformation parameters estimation, respectively. Note that just the scale rate is the parameter where the two approaches agree relatively well $(0.03 \mathrm{ppb} / \mathrm{yr}$ which corresponds to $0.19 \mathrm{~mm} / \mathrm{yr}$ on the Equator). On the other hand the translations show a significant discrepancy which in the case of the $y$ axis reaches $1.05 \mathrm{~mm} / \mathrm{yr}$. It is also worth to mention that we found relatively large orientation discrepancies $(0.83 \mathrm{~mm} / \mathrm{yr}$ for the $x$ axis $)$, while in the comparison between ITRF2008 and DTRF2008 by the Helmert transformation Seitz et al. (2012) estimate $0.4 \mathrm{~mm} / \mathrm{yr}$ for the SLR TRF. The uncertainties of the differences between VEDA and Helmert are at the level of $0.05-0.07 \mathrm{~mm} / \mathrm{yr}$. Hence, 
the differences between the two approaches are statistically significant (the signal to noise ratios are relatively large). This practically means that there are some relative biases (e.g. different core stations selection or/and different adjustment approaches) which the classical Helmert transformation ignores, while the new approach seems to show greater sensitivity. We imply that the VEDA and the classical Helmert transformation show an inconsistency at the level of $1.0 \mathrm{~mm} / \mathrm{yr}$.

The optimal part of the velocities depicts the real velocities which are independent from the reference system and deviate from the TRP velocities. In fact, this is not a problem for the global TRFs, since their main scope is to offer reliable velocities. The global TRF velocities have no particular physical meaning and they refer to a NNR frame which compromises in an optimal way the global tectonic plate motion. Thereafter, the dedicated users (e.g. geophysists, local or continental agencies) can apply the global tectonic plate models (there are plenty of them) in order to derive velocities which have a physical meaning (with respect to a particular tectonic plate).

\section{SUMMARY AND REMARKS}

The VEDA methodology can serve as an alternative diagnostic tool for the assessment of the temporal evolution of the reference frames. The core of the VEDA is the separation (in a least squares sense) of the velocities into two parts: The one part includes the optimal velocities and the other which is called TRP and is related to the geometric effect. The term geometric includes both the systematic effects and the impact of the stations set distribution used for VEDA application. The VEDA is applicable only in relative sense (comparison of two TRFs). It can be applied simultaneously with the classical Helmert transformation. The associated mathematical equations provide a rigorous approach for the assessment of any terrestrial frame. It can also detect systematic effects which the classical Helmert transformation fails to identify, as we find for the case of the ITRF2008 and DTRF2008 comparison. This is achieved due to the ability of handling each reference system separately, unlike the Helmert transformation which deals with the velocity differences.

The VEDA is applied to the assessment of the global TRFs: ITRF2008 and DTRF2008. We found inconsistencies reaching $0.97 \mathrm{~mm} / \mathrm{yr}$ for the translation rates, $0.83 \mathrm{~mm} / \mathrm{yr}$ for the orientation rates and $0.05 \mathrm{ppb} / \mathrm{yr}(0.32 \mathrm{~mm} / \mathrm{yr}$ for the Equator radius $)$ for the scale rates, respectively. Generally, we can infer that the two global TRFs show an agreement at the level of $0.66 \mathrm{~mm} / \mathrm{yr}$ in terms of the datum related parameters. The new methodology should always carefully be applied to "high quality" stations in order to ensure the reliability of the results.

The comparison of our approach and the Helmert transformation shows that the temporal variations of ITRF2008 and DTRF2008 agree at the level of $1.0 \mathrm{~mm} / \mathrm{yr}$. particularly, the largest difference is found in the $y$-axis translation and reaches $1.06 \mathrm{~mm} / \mathrm{yr}$. The differences reach $0.83 \mathrm{~mm} / \mathrm{yr}$ for the $x$ axis orientation and $0.20 \mathrm{~mm} / \mathrm{yr}$ for the scale rate, respectively. The discrepancies between the two methodologies are caused either by the different adjustment strategies and/or different velocity estimation methodologies or/and the different biases handling.

Regarding the optimal velocities differences which play a crucial role for the detection of relative systematic effects, some interesting results are presented. The relative systematic effect is $0.44 \mathrm{~mm} / \mathrm{yr}$ in $3 \mathrm{D}$, while for the vertical part of the velocities it is $0.40 \mathrm{~mm} / \mathrm{yr}$ and for the horizontal one $1.38 \mathrm{~mm} / \mathrm{yr}$.

\section{ACKNOWLEDGMENTS}

The comments and suggestions of Professor Emeritus Athanasios Dermanis led to a significant improvement of the manuscript. Therefore, he is kindly acknowledged. Msc. Dipl. Ing. Nikolaos Demirtzoglou, Dr.Ing. Ilias Daras, Dr.Ing- Christian Gruber, Dr. Ing, Grigorios Tsinidis, MSc. Dipls Ings. Tilemachos Papazois and Evarestos-Theaititos Tentonis gave some valuable hints and comments.

\section{REFERENCES}

Altamimi, Z., Sillard, P. and Boucher, C.: 2002, ITRF2000; $A$ new release of the International Terrestrial Reference Frame for earth science applications. J. Geophys. Res., 107, B10, ETG 2-1-ETG 2-19. DOI: $10.1029 / 2001$ JB000561

Altamimi, Z., Sillard, P. and Boucher, C.: 2003, The impact of a No-Net-Rotation Condition on ITRF2000. Geophys. Res. Lett., 30, 2, 36-1-36-4. DOI: 0.1029/2002GL016279

Altamimi, Z., Collilieux, X., Legrand, J., Garayt, B. and Boucher, C.: 2007, ITRF2005: A new release of the International Terrestrial Reference Frame based on time series of station positions and Earth Orientation Parameters. J. Geophys. Res.: Solid Earth, 112, B9, B09401. DOI: 10.1029/2007JB004949

Altamimi, Z., Collilieux, X. and Boucher, C.: 2008, Accuracy assessment of the ITRF datum definition. In: VI Hotine-Marussi Symposium on Theoretical and Computational Geodesy, IAG Symposia, 132, 101110. DOI: $10.1007 / 978-3-540-74584-6 \_16$

Altamimi, Z. and Dermanis, A: 2009, The choice of reference system in ITRF formulation. In: Sneeuw, N. et al. (eds.), VII Hotine-Marussi Symposium on Mathematical Geodesy, IAG Symposia, 137, 329-334, Springer, Berlin.

DOI: 10.1007/978-3-642-22078-4_49

Altamimi, Z., Collilieux, X. and Métivier, L.: 2011, ITRF2008: An improved solution of the international terrestrial reference frame. J. Geodesy, 85, 8, 457473. DOI: $10.1007 / \mathrm{s} 00190-011-0444-4$

Altamimi, Z., Métivier, L. and Collilieux, X.: 2012, ITRF2008 plate motion model. J. Geophys. Res., 117, B07402. DOI:10.1029/2011JB008930

Altamimi, Z. and Dermanis, A.: 2012, The Choice of Reference System in ITRF Formulation, VII HotineMarussi Symposium on Mathematical Geodesy: Proceedings of the Symposium in Rome, 6-10 June, 2009, International Association of Geodesy Symposia, 137, 329-334, DOI: 10.1007/978-3-642-22078-4_49 
Altamimi, Z. and Dermanis, A.: 2013, Theoretical foundations of ITRF determination. The algebraic and the kinematic approach. In: Katsampalos, K.V., Rossikopoulos, D., Spatalas, S. and Tokmakidis, K. (Eds.), On measurements of lands and constructions. Volume in honor of Prof. Dimitios G. Vlachos. Publication of the School of Rural \& Surveying Engineering, Aristotle University of Thessaloniki, 331-359.

Altamimi, Z., Rebischung, P., Métivier, L. and Collilieux, X.: 2016, ITRF2014: A new release of the International Terrestrial Reference Frame modeling nonlinear station motions. J. Geophys. Res. Solid Earth, 121, 6109-6131. DOI:10.1002/2016JB013098

Angermann, D., Drewes, H., Krügel, M., Meisel, B., Gerstl, M., Kelm, R., Müller, H., Seemüller, W. and Tesmer, V.: 2004, ITRS Combination Centre at DGFI A terrestrial reference frame realization 2003. Deutsche Geodätische Kommission, Reihe B, Nr. 313, München.

Argus, D.F.: 2007, Defining the translational velocity of the reference frame of Earth. Geophys. J. Int., 169, 3, 830-838. DOI:10.1111/j.1365-246X.2007.03344.x

Argus, D.F.: 2012, Uncertainty in the velocity between the mass center and surface of Earth. J. Geophys. Res., 117, B10405. DOI: 10.1029/2012JB009196

Beutler, G., Pearlman, M., Plag, H.-P., Neilan, R., Rothacher, M., and Rummel, R.: 2009, Towards GGOS in 2020. In: Plag, H.-P. and Pearlman, M. (eds.), Global geodetic observing system: meeting the requirements of a global society on a changing planet in 2020, Springer, 273-281. DOI: $10.1007 / 978-3-642-02687-410$

Blewitt, G.: 2003, Self-consistency in reference frames, geocenter definition, and surface loading of the solid Earth. J. Geophys. Res., 108, B2. DOI: $10.1029 / 2002 J B 002082$

Chatzinikos, M. and Dermanis, A.: 2017, A coordinateinvariant model for deforming geodetic networks: understanding rank deficiencies, non-estimability of parameters, and the effect of the choice of minimal constraints. J. Geodesy, 91, 4, 375-396. DOI: $10.1007 / \mathrm{s} 00190-016-0970-1$

Collilieux, X., Altamimi, Z., Ray, J., Van Dam, T. and Wu, X.: 2009, Effect of the satellite laser ranging network distribution on geocenter motion estimation. J. Geophys. Res., Solid Earth, 114. DOI:10.1029/2008JB005727

Collilieux, X. and Altamimi, Z.: 2013, External evaluation of the origin and the scale of the International Terrestrial Reference Frame. In: Altamimi, Z. and Collilieux, X. (eds.), Proceedings of the IAG Symposium REFAG2010, IAG Symposia, 138, 2731. DOI: 10.1007/978-3-642-32998-2 5

Collilieux, X., Altamimi, Z., Argus, D.F., Boucher, C., Dermanis, A., Haines, B.J., Herring, T.A., Kreemer, C., Lemoine, F.G., Ma, C., MacMillan, D.S., Mäkinen, J., Métivier, L., Ries, J.C., Teferle, F. N. and Wu, X.: 2014, External evaluation of the terrestrial reference frame: Report of the task force of the IAG sub-commission 1.2. In: Willis, P. (ed.), Proceedings of the XXV IUGG General Assembly, IAG Symposia, 139. DOI: $10.1007 / 978-3-642-37222-325$

DeMets, C., Gordon, R. G., Argus, D. F. and Stein, S.: 1994, Effect of recent revisions to the geomagnetic reversal time scale on estimates of current plate motions. Geophys. Res. Lett., 21, 20, 2191-2194. DOI: 10.1029/94GL02118
Dermanis, A.: 2001, Global Reference Frames: Connecting observation to theory and geodesy to geophysics. IAG 2001 Scientific Assembly "Vistas for Geodesy in the New Milennium", 2-8 Sept. 2001, Budapest, Hungary.

Dermanis, A.: 2015, Personal communication.

Dermanis, A.: 2019a, Theory and realization of reference systems. In: Freeden, W. and Rummel, R. (eds.), Handbook of Geodesy, Springer Verlag, Heidelberg, Berlin, (in print).

Dermanis, A.: 2019b, Personal communication.

Dong, D.: 2003, Origin of the International Terrestrial Reference Frame. J. Geophys. Res., 108, B4. DOI: $10.1029 / 2002 J B 002035$

Dong, D. and Fang, P.: 2007, ITRF origin: Diagnosis of current realization. EGU General Assemply, Vienna, Austria, 15-20 Apr.

Drewes, H: 1982, A geodetic approach for the recovery of global kinematic plate parameters. Bull. Geod., 56, 1, 70-79.

Drewes, H.: 2009, The actual plate kinematic and crustal deformation model APKIM2005 as basis for a nonrotating ITRF. In: Drewes, H. (ed.), Reference Frames, IAG Symposia, 134, 95-99. DOI: 10.1007/978-3-642-00860-3_15

Fritsche, M, Dietrich, R., Rülke, A., Rothacher, M. and Steigenberger, P.: 2010, Low-degree earth deformation from reprocessed GPS observations. GPS Solut, 14, 165-175. DOI: 10.1007/s10291-009-0130-7

Koch, K.-R.: 1987, Parameter Estimation and Hypothesis Testing in Linear Models. Springer, Berlin, 378 pp.

Kreemer, C., Lavallée, D.A., Blewitt, G. and Holt, W.E.: 2006, On the stability of a geodetic no-net-rotation frame and its implication for the International Terrestrial Reference Frame. Geophys. Res. Lett., 33, L17306. DOI:10.1029/2006GL027058

Kreemer, C., Blewitt, G. and Klein, E.C.: 2014, A geodetic plate motion and Global Strain Rate Model. Geochem.Geophys. Geosyst.,15, 10, 3849-3889. DOI: $10.1002 / 2014 \mathrm{GC} 005407$

Métivier, L., Collilieux, X. and Altamimi, Z.: 2012, ITRF2008 contribution to glacial isostatic adjustment and recent ice melting assessment. Geophys. Res. Lett., 39, L01309. DOI: 10.1029/2011GL049942

Peltier, W. R.: 1998, Postglacial variations in the level of the sea: Implications for climate dynamics and solid-Earth geophysics. Rev. Geophys., 36, 4, 603-689. DOI: $10.1029 / 98 R G 02638$

Petit, G. and Luzum, B. (eds.): 2010, IERS conventions. (IERS Technical Note No. 36), Frankfurt am Main,Verlag des Bundesamts für Kartographie und Geodäsie, 179 pp.

Seitz, M., Angermann, D., Blossfeld, M., Drewes, H. and Gerstl, M.: 2012a, The 2008 DGFI realization of the ITRS: DTRF2008. J. Geodesy, 86, 12, 1097-1123. DOI: $10.1007 / \mathrm{s} 00190-012-0567-2$

Seitz, M., Angermann, D., Blossfeld, M., Drewes, H. and Gerstl, M.: 2012b, The 2008 DGFI realization of the ITRS: DTRF2008 (data). Deutsches Geodätisches Forschungsinstitut, Munich. DOI:10.1594/PANGAEA.834714

Seitz, M., Angermann, D. and Drewes, H.: 2013, Accuracy assessment of ITRS 2008 realization of DGFI: DTRF2008. In: Altamimi, Z. and Collilieux, X. (eds.), Reference frames for applications in geosciences, IAG Symposia, vol. 138, 87-93.

DOI: $10.1007 / 978-3-642-32998-2 \_15$ 
Wu, X., Collilieux, X., Altamimi, Z., Vermeersen, B.L.A., Gross, R.S. and Fukumori, I.: 2011, Accuracy of the international Terrestrial Reference Frame origin and Earth expansion. Geophys. Res. Lett., 38, 13. DOI:10.1029/2011GL047450

Wu, X., Ray, J. and van Dam, T.: 2012, Geocenter motion and its geodetic and geophysical implications. J. Geodyn., 58, 44-61. DOI:10.1016/j.jog.2012.01.007

Wu, X., Abbondanza, C., Altamimi, Z., Chin, T.M., Collilieux, X., Gross, R.S.M., Heflin, B., Jiang, Y. and Parker, J.W.: 2015, KALREF- A Kalman filter and time series approach to the International Terrestrial Reference Frame realization. J. Geophys. Res. Solid Earth, 120, 5, 3775-3802.

DOI: $10.1002 / 2014 J B 011622$
Web

http://itrf.ign.fr/ITRF_solutions/2014_ (accessed 27/04/2016)

\section{APPENDIX: THE OPTIMAL CHOICE OF THE WEIGHT MATRIX}

Let us express the classical least squares solution for the estimated parameters as a function of an arbitrary weigr matrix $\mathbf{W}$ :

$\dot{\boldsymbol{\theta}}_{\text {opt }}=\left(\mathbf{E}^{T} \mathbf{W E}\right)^{-1} \mathbf{E}^{T} \mathbf{W} \mathbf{v}^{\prime}$

The associated covariance matrix will be:

$\mathbf{C}_{\dot{\theta}_{\text {opt }}}=\left(\mathbf{E}^{T} \mathbf{W E}\right)^{-1} \mathbf{E}^{T} \mathbf{W} \mathbf{C}_{\mathbf{v}^{\prime}}^{-1} \mathbf{W E}\left(\mathbf{E}^{T} \mathbf{W E}\right)^{-1}$

where $\mathbf{C}_{\mathbf{e}}$ the covariance matrix of the observational errors.

We are seeking a weight matrix which minimizes the trace of the covariance matrix:

trace $_{\dot{\boldsymbol{\theta}}_{\text {opt }}}(\mathbf{W})=\min$ !

Proof

$\operatorname{trace} \mathbf{C}_{\dot{\boldsymbol{\theta}}}(\mathbf{W})=\min$ ! when:

$\frac{\partial \operatorname{trace} \mathbf{C}_{\dot{\boldsymbol{\theta}}_{\text {opt }}}(\mathbf{W})}{\partial W_{j k}}=\operatorname{trace} \frac{\partial \mathbf{C}_{\dot{\boldsymbol{\theta}}_{o p t}}(\mathbf{W})}{\partial W_{j k}}=0, \quad \forall j, k$

$\operatorname{trace}_{\hat{\hat{\boldsymbol{\theta}}}}(\mathbf{W})=\operatorname{trace}\left[\left(\mathbf{E}^{T} \mathbf{W E}\right)^{-1} \mathbf{E}^{T} \mathbf{W} \mathbf{C}_{\mathbf{e}} \mathbf{W E}\left(\mathbf{E}^{T} \mathbf{W E}\right)^{-1}\right]$

$=\operatorname{trace}\left[\left(\mathbf{E}^{T} \mathbf{W E}\right)^{-2} \mathbf{E}^{T} \mathbf{W} \mathbf{C}_{\mathbf{e}} \mathbf{W E}\right]$

$=\operatorname{trace}(\mathbf{Q})$

where $\mathbf{Q}=\left(\mathbf{E}^{T} \mathbf{W E}\right)^{-2} \mathbf{E}^{T} \mathbf{W} \mathbf{C}_{\mathbf{v}^{\prime}} \mathbf{W E}=\mathbf{N}^{-2} \mathbf{E}^{T} \mathbf{W} \mathbf{C}_{\mathbf{v}^{\prime}} \mathbf{W E}, \mathbf{N}=\mathbf{E}^{T} \mathbf{W E}$.

We seek the weight matrix $\mathbf{W}$ which satisfies:

$\operatorname{trace} \frac{\partial \mathbf{Q}}{\partial W_{j k}}=0, \quad \forall j, k$

Recalling that:

$\frac{\partial \mathbf{W}}{\partial W_{j k}}=\mathbf{i}_{j} \mathbf{i}_{k}^{T}$

where $\mathbf{i}_{k}$ is the $\mathrm{k}^{\text {th }}$ of the $n \times \mathrm{x} n$ identity matrix $\mathbf{I}_{n}=\left[\begin{array}{lll}\mathbf{i}_{j} & \cdots & \mathbf{i}_{k}\end{array}\right]$. Taking into account that $\mathbf{N}^{-2} \mathbf{N}^{2}=\mathbf{I}$ it follows thc (Dermanis, 2015): 


$$
\begin{aligned}
\mathbf{0} & =\frac{\partial \mathbf{I}}{\partial l}=\frac{\partial \mathbf{N}^{-2} \mathbf{N}^{2}}{\partial l}=\frac{\partial \mathbf{N}^{2}}{\partial l} \mathbf{N}^{-2}+\frac{\partial \mathbf{N}^{-2}}{\partial l} \mathbf{N}^{2} \Rightarrow \\
\mathbf{0} & =\left(\frac{\partial \mathbf{N}}{\partial l} \mathbf{N}+\mathbf{N} \frac{\partial \mathbf{N}}{\partial l}\right) \mathbf{N}^{-2}+\mathbf{N}^{2} \frac{\partial \mathbf{N}^{-2}}{\partial l} \Rightarrow \\
\mathbf{0} & =\frac{\partial \mathbf{N}}{\partial l} \mathbf{N}^{-1}+\mathbf{N} \frac{\partial \mathbf{N}}{\partial l} \mathbf{N}^{-2}+\mathbf{N}^{2} \frac{\partial \mathbf{N}^{-2}}{\partial l}
\end{aligned}
$$

and hence:

$$
\frac{\partial \mathbf{N}^{-2}}{\partial l}=-\mathbf{N}^{-2} \frac{\partial \mathbf{N}}{\partial l} \mathbf{N}^{-1}-\mathbf{N}^{-1} \frac{\partial \mathbf{N}}{\partial l} \mathbf{N}^{-2}
$$

Thus:

$$
\frac{\partial \mathbf{N}}{\partial W_{j k}}=\mathbf{E}^{T} \frac{\partial \mathbf{W}}{\partial W_{j k}} \mathbf{E}=\mathbf{E}^{T} \mathbf{i}_{j} i_{k}^{T} \mathbf{E}
$$

so that:

$$
\begin{aligned}
& \frac{\partial \mathbf{N}^{-2}}{\partial W_{j k}}=-\frac{\partial \mathbf{N}}{\partial W_{j k}} \mathbf{N}^{-1}-\mathbf{N}^{-1} \frac{\partial \mathbf{N}}{\partial W_{j k}} \mathbf{N}^{-2} \Rightarrow \\
& \frac{\partial \mathbf{N}^{-2}}{\partial W_{j k}}=\mathbf{E}^{T} \mathbf{i}_{j} \mathbf{i}_{k}^{T} \mathbf{E} \mathbf{N}^{-1}-\mathbf{N}^{-1} \mathbf{E}^{T} \mathbf{i}_{j} \mathbf{i}_{k}^{T} \mathbf{E} \mathbf{N}^{-2}
\end{aligned}
$$

and

$\frac{\partial \mathbf{Q}}{\partial W_{j k}}=-\mathbf{N}^{-2} \mathbf{E}^{T} \mathbf{W} \mathbf{C}_{\mathbf{v}^{\prime}} \mathbf{W E}+\mathbf{N}^{-2} \mathbf{E}^{T} \frac{\partial \mathbf{W}}{\partial W_{j k}} \mathbf{C}_{\mathbf{e}} \mathbf{W E}+\mathbf{N}^{-2} \mathbf{E}^{T} \mathbf{W} \mathbf{C}_{\mathbf{v}^{\prime}} \mathbf{i}_{j} \mathbf{i}_{k}^{T} \mathbf{E} \Rightarrow$

$\frac{\partial \mathbf{Q}}{\partial W_{j k}}=\left[-\mathbf{N}^{-2} \mathbf{E}^{T} \mathbf{i}_{j} \mathbf{i}_{k}^{T} \mathbf{E} \mathbf{N}^{-1}-\mathbf{N}^{-1} \mathbf{E}^{T} \mathbf{i}_{j} \mathbf{i}_{k}^{T} \mathbf{E N}^{-2}\right] \mathbf{E}^{T} \mathbf{W} \mathbf{C}_{\mathbf{v}^{\prime}} \mathbf{E}+\mathbf{N}^{-2} \mathbf{E}^{T} \mathbf{i}_{j} \mathbf{i}_{k}^{T} \mathbf{C}_{\mathbf{v}^{\prime}} \mathbf{W E}+\mathbf{N}^{-2} \mathbf{E}^{T} \mathbf{W} \mathbf{C}_{\mathbf{v}} \mathbf{i}_{j} \mathbf{i}_{k}^{T} \mathbf{E}$

Therefore

$$
\begin{aligned}
& 0=\frac{\partial \operatorname{trace} \mathbf{C}_{\dot{\boldsymbol{\theta}}_{\text {opt }}}(\mathbf{W})}{\partial W_{j k}}=\frac{\partial \operatorname{trace} \mathbf{Q}}{\partial W_{j k}}=\operatorname{trace} \frac{\partial \mathbf{Q}}{\partial W_{j k}} \Rightarrow \\
& 0=\operatorname{trace}\left\{\left[-\mathbf{N}^{-2} \mathbf{E}^{T} \mathbf{i}_{j} \mathbf{i}_{k}^{T} \mathbf{E} \mathbf{N}^{-1}-\mathbf{N}^{-1} \mathbf{E}^{T} \mathbf{i}_{j} \mathbf{i}_{k}^{T} \mathbf{E} \mathbf{N}^{-2}\right] \mathbf{E}^{T} \mathbf{W} \mathbf{C}_{\mathbf{v}^{\prime}} \mathbf{E}+\mathbf{N}^{-2} \mathbf{E}^{T} \mathbf{i}_{j} \mathbf{i}_{k}^{T} \mathbf{C}_{\mathbf{v}^{\prime}} \mathbf{W} \mathbf{E}+\mathbf{N}^{-2} \mathbf{E}^{T} \mathbf{W} \mathbf{C}_{\mathbf{v}^{\prime}} \mathbf{i}_{j} \mathbf{i}_{k}^{T} \mathbf{E}\right\} \Rightarrow \\
& 0=\operatorname{trace}\left\{-\mathbf{N}^{-2} \mathbf{E}^{T} \mathbf{i}_{j} \mathbf{i}_{k}^{T} \mathbf{E} \mathbf{N}^{-1} \mathbf{E}^{T} \mathbf{W} \mathbf{C}_{\mathbf{v}^{\prime}} \mathbf{E}-\mathbf{N}^{-1} \mathbf{E}^{T} \mathbf{i}_{j} \mathbf{i}_{k}^{T} \mathbf{E} \mathbf{N}^{-2} \mathbf{E}^{T} \mathbf{W} \mathbf{C}_{\mathbf{v}} \mathbf{E}+\mathbf{N}^{-2} \mathbf{E}^{T} \mathbf{i}_{j} \mathbf{i}_{k}^{T} \mathbf{C}_{\mathbf{v}^{\prime}} \mathbf{W E}+\mathbf{N}^{-2} \mathbf{E}^{T} \mathbf{W} \mathbf{C}_{\mathbf{v}^{\prime}} \mathbf{i}_{j} \mathbf{i}_{k}^{T} \mathbf{E}\right\} \Rightarrow \\
& 0=\operatorname{trace}\left\{-\mathbf{i}_{k}^{T} \mathbf{E N}^{-1} \mathbf{E}^{T} \mathbf{W} \mathbf{C}_{\mathbf{v}^{\prime}} \mathbf{W} \mathbf{E} \mathbf{N}^{-2} \mathbf{E}^{T} \mathbf{i}_{j}-\mathbf{i}_{k}^{T} \mathbf{E N}^{-2} \mathbf{E}^{T} \mathbf{W} \mathbf{C}_{\mathbf{v}^{\prime}} \mathbf{W} \mathbf{E} \mathbf{N}^{-1} \mathbf{E}^{T} \mathbf{i}_{j}+\mathbf{i}_{k}^{T} \mathbf{C}_{\mathbf{v}^{\prime}} \mathbf{W} \mathbf{E} \mathbf{N}^{-2} \mathbf{E}^{T} \mathbf{i}_{j}+\mathbf{i}_{k}^{T} \mathbf{E N}^{-2} \mathbf{E}^{T} \mathbf{W C}_{\mathbf{v}^{\prime}} \mathbf{i}_{j}\right\} \Rightarrow \\
& 0=\operatorname{trace}\left\{-\mathbf{E N}^{-1} \mathbf{E}^{T} \mathbf{W} \mathbf{C}_{\mathbf{v}^{\prime}} \mathbf{W} \mathbf{E} \mathbf{N}^{-2} \mathbf{E}^{T}-\mathbf{E N}^{-2} \mathbf{E}^{T} \mathbf{W} \mathbf{C}_{\mathbf{v}^{\prime}} \mathbf{W} \mathbf{E} \mathbf{N}^{-1} \mathbf{E}^{T}+\mathbf{C}_{\mathbf{v}^{\prime}} \mathbf{W} \mathbf{E} \mathbf{N}^{-2} \mathbf{E}^{T}+\mathbf{E} \mathbf{N}^{-2} \mathbf{E}^{T} \mathbf{W C}_{\mathbf{v}^{\prime}}\right\}_{k j}
\end{aligned}
$$

We are now seeking a solution to

$\mathbf{L}(\mathbf{W})=-\mathbf{E} \mathbf{N}^{-1} \mathbf{E}^{T} \mathbf{W} \mathbf{C}_{\mathbf{v}}, \mathbf{W} \mathbf{E} \mathbf{N}^{-2} \mathbf{E}^{T}-\mathbf{E} \mathbf{N}^{-2} \mathbf{E}^{T} \mathbf{W} \mathbf{C}_{\mathbf{v}}, \mathbf{W} \mathbf{E} \mathbf{N}^{-1} \mathbf{E}^{T}+\mathbf{C}_{\mathrm{e}} \mathbf{W E N} \mathbf{N}^{-2} \mathbf{E}^{T}+\mathbf{E} \mathbf{N}^{-2} \mathbf{E}^{T} \mathbf{W} \mathbf{C}_{\mathbf{v}^{\prime}}=0$

Assuming that $\mathbf{W}=\mathbf{C}_{\mathbf{v}^{\prime}}^{-1}$ we get:

$$
\begin{aligned}
& \mathbf{L}\left(\mathbf{C}_{v^{\prime}}^{-1}\right)=-\mathbf{E} \mathbf{N}^{-1} \mathbf{E}^{T} \mathbf{C}_{v^{\prime}}^{-1} \mathbf{C}_{v^{\prime}} \mathbf{C}_{v^{\prime}}^{-1} \mathbf{E} \mathbf{N}^{-2} \mathbf{E}^{T}-\mathbf{E} \mathbf{N}^{-2} \mathbf{E}^{T} \mathbf{C}_{v^{\prime}}^{-1} \mathbf{C}_{v^{\prime}} \mathbf{C}_{v^{\prime}}^{-1} \mathbf{E} \mathbf{N}^{-1} \mathbf{E}^{T}+\mathbf{C}_{v^{\prime}} \mathbf{C}_{v^{\prime}}^{-1} \mathbf{E} \mathbf{N}^{-2} \mathbf{E}^{T}+\mathbf{E} \mathbf{N}^{-2} \mathbf{E}^{T} \mathbf{C}_{v^{\prime}}^{-1} \mathbf{C}_{v^{\prime}} \Rightarrow \\
& \mathbf{L}\left(\mathbf{C}_{v^{\prime}}^{-1}\right)=-\mathbf{E} \mathbf{N}^{-1} \mathbf{E}^{T} \mathbf{C}_{v^{\prime}}^{-1} \mathbf{E} \mathbf{N}^{-2} \mathbf{E}^{T}-\mathbf{E} \mathbf{N}^{-2} \mathbf{E}^{T} \mathbf{C}_{v^{\prime}}^{-1} \mathbf{E N}^{-1} \mathbf{E}^{T}+\mathbf{E} \mathbf{N}^{-2} \mathbf{E}^{T}+\mathbf{E} \mathbf{N}^{-2} \mathbf{E}^{T}
\end{aligned}
$$

Taking into account that $\mathbf{N}=\mathbf{E}^{T} \mathbf{C}_{\mathrm{e}}^{-1} \mathbf{E}$, Equation (A15), becomes:

$$
\mathbf{L}\left(\mathbf{C}_{\mathrm{e}}^{-1}\right)=-\mathbf{E N}^{-1} \mathbf{N} \mathbf{N}^{-2} \mathbf{E}^{T}-\mathbf{E N}^{-2} \mathbf{N} \mathbf{N}^{-1} \mathbf{E}^{T}+\mathbf{E N}^{-2} \mathbf{E}^{T}+\mathbf{E} \mathbf{N}^{-2} \mathbf{E}^{T}=\mathbf{0}
$$

Thus, the choice of the inverse of the covariance of the observational errors as the weight matrix, leads to the minimization of the trace of the associated covariance matrix of the estimated parameters. 